\title{
A Safe Transitions Pathway for post-craniotomy neurological surgery patients: high-value care that bypasses the intensive care unit
}

\author{
Jacob S. Young, MD, ${ }^{1}$ Andrew K. Chan, MD, ${ }^{1}$ Jennifer A. Viner, NP, ${ }^{1}$ Sujatha Sankaran, MD, ${ }^{2}$ \\ Alvin Y. Chan, MD, ${ }^{3}$ Sarah Imershein, MPH, ${ }^{4}$ Aldea Meary-Miller, MBA, MPH, ${ }^{4}$ \\ Philip V. Theodosopoulos, MD, ${ }^{1}$ Line Jacques, MD, ${ }^{1}$ Manish K. Aghi, MD, PhD, ${ }^{1}$ \\ Edward F. Chang, MD, ${ }^{1}$ Shawn L. Hervey-Jumper, MD, ${ }^{1}$ Tracy Ward, NP, Liz Gibson, NP, ${ }^{1}$ \\ Mariann M. Ward, NP, MS, ${ }^{1}$ Peter Sanftner, NP, ${ }^{1}$ Stacy Wong, NP, ${ }^{1}$ Dominic Amara, BA, ${ }^{5}$ \\ Stephen T. Magill, MD, PhD, ${ }^{1}$ Joseph A. Osorio, MD, PhD, ${ }^{1}$ Brinda Venkatesh, MPH, ${ }^{4}$ \\ Ralph Gonzales, MD, ${ }^{5}$ Catherine Lau, MD, ${ }^{2}$ Christy Boscardin, PhD, ${ }^{5}$ Michael Wang, ${ }^{4}$ \\ Kim Berry, MBA, ${ }^{4}$ Laurie McCullagh, RN, ${ }^{4}$ Mary Reid, RN, ${ }^{4}$ Kayla Reels, RN, ${ }^{4}$ Sara Nedkov, RN, ${ }^{4}$ \\ Mitchel S. Berger, MD, ${ }^{1}$ and Michael W. McDermott, MD'
}

\begin{abstract}
1Department of Neurological Surgery and 2Division of Hospital Medicine, Department of Medicine, University of California, San Francisco; ${ }^{3}$ Department of Neurological Surgery, University of California, Irvine; ${ }^{4}$ University of California San Francisco Medical Center, San Francisco; and ${ }^{5}$ School of Medicine, University of California, San Francisco, California
\end{abstract}

\begin{abstract}
OBJECTIVE High-value medical care is described as care that leads to excellent patient outcomes, high patient satisfaction, and efficient costs. Neurosurgical care in particular can be expensive for the hospital, as substantial costs are accrued during the operation and throughout the postoperative stay. The authors developed a "Safe Transitions Pathway" (STP) model in which select patients went to the postanesthesia care unit (PACU) and then the neuro-transitional care unit (NTCU) rather than being directly admitted to the neurosciences intensive care unit (ICU) following a craniotomy. They sought to evaluate the clinical and financial outcomes as well as the impact on the patient experience for patients who participated in the STP and bypassed the ICU level of care.
\end{abstract}

METHODS Patients were enrolled during the 2018 fiscal year (FY18; July 1, 2017, through June 30, 2018). The electronic medical record was reviewed for clinical information and the hospital cost accounting record was reviewed for financial information. Nurses and patients were given a satisfaction survey to assess their respective impressions of the hospital stay and of the recovery pathway.

RESULTS No patients who proceeded to the NTCU postoperatively were upgraded to the ICU level of care postoperatively. There were no deaths in the STP group, and no patients required a return to the operating room during their hospitalization $(95 \% \mathrm{Cl} 0 \%-3.9 \%)$. There was a trend toward fewer 30-day readmissions in the STP patients than in the standard pathway patients $(1.2 \%[95 \% \mathrm{Cl} 0.0 \%-6.8 \%]$ vs $5.1 \%[95 \% \mathrm{Cl} 2.5 \%-9.1 \%], \mathrm{p}=0.058)$. The mean number of ICU days saved per case was 1.20 . The average postprocedure length of stay was reduced by 0.25 days for STP patients. Actual FY18 direct cost savings from 94 patients who went through the STP was $\$ 422,128$.

CONCLUSIONS Length of stay, direct cost per case, and ICU days were significantly less after the adoption of the STP, and ICU bed utilization was freed for acute admissions and transfers. There were no substantial complications or adverse patient outcomes in the STP group.

https://thejns.org/doi/abs/10.3171/2020.3.JNS192133

KEYWORDS safety; quality; cost-saving; postoperative recovery; craniotomy; oncology

ABBREVIATIONS CMI = case mix index; DVT = deep venous thrombosis; FY18 = 2018 fiscal year; ICU = intensive care unit; $L O S=$ length of stay; MVD = microvascular decompression; NTCU = neuro-transitional care unit; PACU = postanesthesia care unit; PE = pulmonary embolism; SSI = surgical site infection; STP = Safe Transitions Pathway; UTI = urinary tract infection.

SUBMITTED August 8, 2019. ACCEPTED March 11, 2020.

INCLUDE WHEN CITING Published online May 29, 2020; DOI: 10.3171/2020.3.JNS192133. 
I NTENSIVE care unit (ICU) stays are costly for patients and the hospital. ${ }^{1,2}$ As such, reducing ICU length of stay (LOS) and overall admission times are important metrics for lowering hospital costs. ${ }^{3}$ At our institution, patients undergoing craniotomy for supratentorial brain tumor resection, Chiari malformation decompression, or microvascular decompression (MVD) historically have recovered in the ICU postoperatively for close observation. Hourly neurological evaluations and vital sign monitoring, as well as continuous cardiac monitoring and pulse oximetry, were thought to be necessary to identify postoperative complications quickly. ${ }^{4}$ However, placing patients in the ICU following a craniotomy occupies beds, which can limit operative case volume when there is no bed availability in the ICU for patients requiring postoperative care. Furthermore, automatically placing all post-craniotomy patients in the ICU following surgery limits the institution's ability to accept transfers of acute patients from community hospitals and emergency room admissions.

Despite this routine use of ICU care for all patients who undergo craniotomy, not all of these patients are at high risk for neurological decline. While it is well established that some procedures are riskier than others, identifying the patient factors that increase the risk for postoperative decline is a source of debate and controversy. ${ }^{5,6}$ One retrospective analysis of 400 adult patients at Massachusetts General Hospital who underwent craniotomy during the period from April 2010 to March 2011 showed that patients with diabetes mellitus, high intraoperative blood loss, and longer surgical procedures were at a higher risk for requiring an ICU level of postoperative care. ${ }^{7}$ In addition, the authors found that only $140(35.0 \%)$ of 400 patients actually required an ICU level of care and that only $33(8.3 \%)$ required care other than intravenous medications for blood pressure control. It is unclear whether patients who are otherwise healthy, have small structural brain lesions, and/ or undergo short operations can bypass the ICU and transition directly to a lower level of care postoperatively.

We developed a Safe Transitions Pathway (STP) at our institution, through which select patients who underwent elective craniotomy for brain tumor resection, MVD, or Chiari malformation decompression, after recovery in the postanesthesia care unit (PACU), were admitted directly to our step-down unit, the neuro-transitional care unit (NTCU), rather than going directly to the ICU postoperatively, which is the standard care path for patients undergoing these procedures. ${ }^{8,9}$ At the NTCU level of care, nursing is staffed at a ratio of 2-3 patients per nurse, compared with 1.5 patients per nurse for ICU care; neurological examinations and vital checks can be performed every 2 hours, compared to hourly in the ICU; and continuous pulse oximetry and cardiac telemetry monitoring are done for 24 hours. By nature of their pathway, STP patients had no ICU days during their admission. To this end, we retrospectively analyzed clinical and financial outcomes for STP patients to determine the safety and utility, as well as the cost-effectiveness, of this model.

\section{Methods \\ Patient Selection}

The following criteria were used to help with initial patient selection: 1 ) patient age $\leq 65$ years and no premorbid conditions that would otherwise necessitate ICU-level care (such as significant cardiac or pulmonary disease); 2) planned routine craniotomy and, if a brain tumor case, the tumor must be supratentorial in location and $<3 \mathrm{~cm}$ in size; other patient populations included those undergoing Chiari malformation decompression or microvascular decompression for trigeminal or glossopharyngeal neuralgia and hemifacial spasm; and 3) no intraoperative adverse events, operative time $<5$ hours, estimated blood loss $<$ $500 \mathrm{~mL}$, and a routine extubation.

Enrollment in the STP was decided upon at the discretion of the attending neurosurgeon. All STP patients who otherwise would have had postsurgical care according to the standard ICU care pathway were elective admissions, and $6(6.4 \%)$ STP patients were either transferred to the hospital urgently or admitted from the emergency room $(\mathrm{p}<0.001)$. During the course of the study, as surgeons became more familiar with the STP, criteria expanded to include patients older than 65 years, patients with retrosigmoid craniectomies, and patients with small posterior fossa tumors of the petrous face or cerebellum. For a detailed description of how patients were selected for STP, refer to Osorio et al. ${ }^{8}$

\section{Nursing and Clinical Team Communication}

The unit-based clinical nurse champion delivered educational in-service training sessions on this new patient population to the NTCU and PACU nursing staffs. To help set patient expectations, trifold patient information guides for each STP procedure type were developed and distributed to patients during preoperative clinic visits. Workflow redesigns and posters helped the neurosurgery attending physicians, residents, nurse practitioners, nurses, and anesthesia team members to identify eligible patient populations and reinforce the expected postoperative monitoring guidelines.

Information technology builds for the electronic medical record (EPIC) were created for dedicated order sets, and eligibility criteria pop-ups were displayed on the computer screen. Weekly case list communications were distributed to all members of the team, including the bed control and charge nurses.

\section{Clinical and Financial Information}

Patients were enrolled in the study during the 2018 fiscal year (FY18; July 1, 2017, through June 30, 2018). The electronic medical record was reviewed for clinical information, and hospital cost accounting records were reviewed for financial information. Outcomes were calculated to reflect LOS differentials, direct cost savings for patients going through the ICU care pathway versus the STP, and any adverse outcomes, including escalation in nursing care and increased 30-day readmission rates. Surveys were distributed for patients and nurses to document their satisfaction with the program.

\section{Statistical Analysis}

All statistical analyses were performed with JMP Pro 14 (SAS Institute Inc.). Pooled t-tests were performed to 
TABLE 1. Patient demographics

\begin{tabular}{lccc}
\hline & STP Pts & Standard Pts & p Value \\
\hline Total no. of pts & 94 & 220 & \\
\hline Tumor pts & $59(62.8 \%)$ & $202(91.8 \%)$ & $<0.001$ \\
\hline MVD pts & $18(19.1 \%)$ & $17(7.7 \%)$ & \\
\hline Chiari pts & $17(18.1 \%)$ & $1(0.5 \%)$ & \\
\hline $\begin{array}{l}\text { Overall mean age in yrs } \\
\text { (range) }\end{array}$ & $49.1(18-81)$ & $46.8(15-65)$ & 0.21 \\
\hline Mean age in yrs $(\%>65)$ & & & \\
\hline Tumor pts & $53.0(20.3 \%)$ & $46.3(0 \%)$ & 0.001 \\
\hline MVD pts & $50.1(11.1 \%)$ & $53.8(0 \%)$ & 0.36 \\
\hline Chiari pts & $34.5(0 \%)$ & $27.0(0 \%)$ & 0.51 \\
\hline Mean CMI, all pts & 3.7 & 4.2 & $<0.001$ \\
\hline Tumor pts & 4.19 & 4.22 & 0.67 \\
\hline MVD pts & 3.08 & 3.48 & 0.22 \\
\hline Chiari pts & 2.74 & 3.00 & 0.44 \\
\hline Sex & & & 0.49 \\
\hline Male & $34.0 \%$ & $38.2 \%$ & \\
\hline Female & $66.0 \%$ & $61.8 \%$ & \\
\hline
\end{tabular}

$\mathrm{Pt}=$ patient.

determine if there was a difference between 2 means, with a threshold of $<0.05$ set to determine statistical significance. The regression model was generated with Stata (StataCorp LP).

\section{Results}

\section{Patient Demographics}

Ninety-four patients went through the STP, compared with 220 patients in the standard pathway. The average age for an STP patient was 49.1 years, compared with 46.8 years for a standard pathway patient $(\mathrm{p}=0.21)$. The standard pathway patients had a higher case mix index (CMI; an acuity metric defined by Medicare and used to adjust costs and outcomes based on billing data) $)^{10}$ than the STP patients $(\mathrm{p}<0.001)$. The average PACU stay for an STP patient was 1 hour and 38 minutes (range 1-4 hours). There were 59 patients $(62.8 \%)$ with cranial tumors who underwent resection, 18 (19.1\%) who had an MVD for cranial neuralgia, and 17 (18.0\%) who underwent Chiari malformation decompression. After initiation of the STP, 94\% of all Chiari patients were enrolled in the STP. For detailed patient demographics, see Table 1.

\section{Safety}

Four patients who were initially planned for the STP did not complete the pathway. One of these patients switched to the standard pathway due to a prolonged operating time; 1 patient had extensive tumor growth found on the preoperative MRI that resulted in a surgical plan that was no longer appropriate for the STP; 1 patient had an intraoperative complication; and for 1 patient the STP destination was not communicated to the operating room anesthesia and nursing team by the neurosurgeons, which resulted in the patient being erroneously admitted to the ICU. No pa-
TABLE 2. Clinical outcomes

\begin{tabular}{cccc}
\hline & STP Pts & Standard Pts & p Value \\
\hline Overall LOS, days & $2.62 \pm 0.29$ & $2.69 \pm 0.11$ & 0.64 \\
\hline Tumor pts & 2.71 & 2.72 & 0.97 \\
\hline MVD pts & 2.22 & 2.41 & 0.44 \\
\hline Chiari pts & 2.71 & 2.00 & 0.54 \\
\hline Postop LOS, days & 1.44 & 1.69 & 0.03 \\
\hline Tumor pts & 1.47 & 1.72 & 0.06 \\
\hline MVD pts & 1.22 & 1.41 & 0.44 \\
\hline Chiari pts & 1.53 & 1.00 & 0.48 \\
\hline Mean ICU LOS, days & 0 & 1.20 & $<0.001$ \\
\hline Tumor pts & 0 & 1.21 & $<0.001$ \\
\hline MVD pts & 0 & 1.12 & $<0.001$ \\
\hline Chiari pts & 0 & 1.00 & $<0.001$ \\
\hline Discharge disposition & \multicolumn{3}{c}{0.07} \\
\hline Home & $87.2 \%$ & $85.5 \%$ & \\
\hline Acute rehab & $1.1 \%$ & $6.8 \%$ & \\
\hline Skilled nursing facility & $1.1 \%$ & $0.5 \%$ & \\
\hline Home healthcare & $10.6 \%$ & $7.3 \%$ & \\
\hline 30-day readmission, all pts & $1.2 \%$ & $5.1 \%$ & 0.058 \\
\hline Tumor pts & $0.0 \%(\mathrm{n}=0)$ & $5.0 \%(\mathrm{n}=9)$ & 0.10 \\
\hline MVD pts & $7.7 \%(\mathrm{n}=1)$ & $5.9 \%(\mathrm{n}=1)$ & 0.97 \\
\hline Chiari pts & $0.0 \%(\mathrm{n}=0)$ & $0.0 \%(\mathrm{n}=0)$ & 0.99 \\
\hline
\end{tabular}

tients who proceeded to the NTCU postoperatively were upgraded to the ICU level of care. Furthermore, there were no deaths among the STP patients and no patient required a return to the operating room during their hospitalization. Additionally, there was a trend toward fewer 30-day readmissions in the STP patients than in the standard pathway patients $(1.2 \%$ vs $5.1 \%, \mathrm{p}=0.058)$. Overall, recovery in the NTCU after a prolonged PACU stay did not negatively impact patient outcomes or safety (see Table 2 for clinical outcomes). Within 30 days of surgery, $2.1 \%$ of patients had a surgical site infection (SSI), $2.1 \%$ had a deep venous thrombosis (DVT) or pulmonary embolism (PE), and $0 \%$ had pneumonia-results comparable to those for patients undergoing similar procedures reported in the published literature. $^{11}$

\section{Direct Cost Savings}

Actual FY18 direct cost savings to the institution's health system from 94 patients who went through the STP were $\$ 422,128$ (for detailed financial results, refer to Table 3 ). In addition to the direct savings, additional revenue is possible for the hospital based on the "creation" of 113 ICU bed-days, admittedly at the expense of transitional care unit (TCU) beds.

\section{Regression Model}

Direct cost savings in the STP group compared to the standard care group were statistically significant $(\mathrm{p}=$ 0.02) after adjusting for CMI, age, procedure type, time variation, and surgeon. Direct cost savings in this gener- 
TABLE 3. Financial outcomes

\begin{tabular}{llllc}
\hline & STP Pts & Standard Pts & Difference & p Value \\
\hline Direct cost & $\$ 16,808.42$ & $\$ 21,299.14$ & $-\$ 4,490.72$ & $<0.001$ \\
\hline Tumor pts & $\$ 18,227$ & $\$ 21,899$ & $-\$ 3,672$ & $<0.001$ \\
\hline MVD pts & $\$ 14,305$ & $\$ 14,537$ & $-\$ 232$ & 0.71 \\
\hline Chiari pts & $\$ 14,536$ & $\$ 15,064$ & $-\$ 528$ & 0.78 \\
\hline
\end{tabular}

alizable model were approximately $\$ 1632$ per case, with an actual cost savings to the hospital of $\$ 4491$ per case for each STP patient.

\section{Length of Stay}

The average postprocedure LOS was reduced by 0.25 days for STP patients $(\mathrm{p}=0.03)$. Additionally, the mean number of ICU days saved per case was 1.20 , for a total of 113 ICU days among 94 STP patients.

\section{Patient Experience}

On a survey distributed to patients, $73 \%(\mathrm{n}=31)$ of patients who underwent craniotomy followed by the STP for immediate postsurgical recovery care rated their overall care and experience as "excellent," compared to $86 \%$ $(\mathrm{n}=29)$ who received care through to the standard ICU pathway (difference $15 \%, 95 \% \mathrm{CI}-6 \%$ to $35 \%$, chi-square $2.58, \mathrm{p}=0.46$ ). Additionally, $55 \%$ of STP patients rated their privacy and noise control as "excellent," compared to $41 \%$ in the standard ICU pathway group (difference 14\%, $95 \% \mathrm{CI}-11 \%$ to $36 \%$, chi-square $2.96, \mathrm{p}=0.56$ ).

\section{Nursing Experience}

Results from the surveys distributed to nurses showed that $87.5 \%(n=8)$ of PACU nurses who completed the survey after implementation of the study reported they were either "very satisfied" or "satisfied" with their experience working with STP patients. There was universal consensus among NTCU nurses $(\mathrm{n}=30)$ that they wanted more neurosurgical patients admitted to the unit, and over $90 \%$ of NTCU nurses reported a "high" or "very high" comfort level working with patients who had surgical subgaleal drains or external ventricular drains in place.

\section{Discussion}

Here we describe the clinical and financial outcomes after implementation of a new Safe Transitions Pathway (STP) for appropriate patients undergoing elective craniotomies for supratentorial brain tumor resections, MVDs, or Chiari malformation decompressions. This study was a follow-up of a previously published pilot study at our institution that showed substantial financial savings with no complications or escalation of care. ${ }^{8}$ The main limitation of that prior study was that there were only 10 patients, and all had undergone supratentorial brain tumor resections. The addition of this new review of the STP expands the indications and increases the number of patients. Our results showed that the adoption of STP could be a costsaving and safe alternative to the default postoperative ICU admissions for all patients undergoing craniotomy.
Indeed, after PACU recovery direct postsurgial admission to the NTCU may be superior to direct ICU admission because patients undergoing care through the STP had fewer complaints related to privacy and noise. Compared with standard path patients, the LOS of STP patients was shorter by a quarter of a day. The actual costs saved for STP patients was over $\$ 4000$ per case, and there were no adverse events. Furthermore, the NTCU and PACU nursing staffs were nearly unanimously in favor of caring for these postoperative patients.

The results of our study are similar to those found in previous studies that show ICU admission may not be required for select patients undergoing elective craniotomies. ${ }^{12}$ Florman et al. found that there were no major complications during the hospital stay when 200 consecutive elective craniotomy and supratentorial tumor resection patients were admitted directly to the neurosurgical floor. ${ }^{12}$ Only $5(2.0 \%)$ of these patients required escalation to transitional-level nursing care within the first 48 hours postoperatively. No patients required transfer to the ICU. In 2016, Venkatraghavan et al. demonstrated that patients with supratentorial tumors who undergo operations shorter than 4 hours and who were placed on an enhanced recovery after surgery pathway may be discharged home on the same day with minimal readmissions or complications. ${ }^{13}$ One limitation of these studies was that only patients who underwent supratentorial tumor resection were included. Moreover, no patient financial data were collected. For the present study, the target population included only patients undergoing tumor resections, MVDs, and Chiari malformation decompressions, but as more surgeons adopted STP, the criteria were then expanded to patients undergoing other procedures, such as retrosigmoid craniotomies for cerebellar lesions and petrous face lesions. These patients showed favorable financial outcomes and clinical outcomes similar to those reported here, with no complications and no patients requiring transfer to the ICU.

Given the potentially devastating consequences that can occur after a craniotomy, such as a postoperative hematoma, there is understandable hesitation regarding early discharge home after craniotomy. However, studies have shown that most serious complications occur within hours, not days, of the procedure. ${ }^{14}$ Moreover, longer admission times would not have prevented the subacute and delayed complications, such as a wound infection or CSF leak, that lead to 30-day readmissions. Since shorter hospital stays are associated with fewer hospital-acquired infections such as urinary tract infections (UTIs) and decreased complications like DVTs and PEs, an added benefit of the STP was shorter LOS, which also resulted in lower costs. ${ }^{15}$

There are multiple advantages to bypassing the ICU in this patient cohort. The risks are likely to be minimal because the complication rate is low, as reported in the literature and demonstrated in the current study. ${ }^{12}$ An unmeasured advantage of admitting patients to the NTCU following relatively routine cranial procedures is that ICU beds are opened up for patients who require the resources and nursing care that can only be provided in the ICU. There were 113 ICU days freed up due to the implementation of the STP. These ICU beds freed by the STP may have 
significant positive revenue implications for the health system. This study demonstrated, on average, $\$ 4491$ direct cost per case savings for a total of $\$ 422,128$ for the year. These savings were generated by enrolling only $29.9 \%$ of the potentially eligible STP patient population in the pathway, which suggests that there are still significant opportunities for additional cost savings from increased case volumes. In the future, as institutional comfort continues to grow with the STP, it is possible that some patients with the lowest immediate postoperative risks could be transferred to the acute-care floor (i.e., with an even higher patient to nurse ratio) following a PACU stay, potentially augmenting cost savings and patient comfort.

As previously mentioned in the report of our pilot study, ${ }^{8}$ a number of factors have made the STP model sustainable. First, the electronic record at our institution has allowed for order sets that make the STP easy to implement. Second, we have expanded the target patient population to also include small extra- and intraaxial tumors of the posterior fossa, MVDs, and Chiari malformation decompressions. The inclusion of these cases increased the volume of patients going through the STP, which provided our NTCU nursing team with more experience in caring for patients earlier in their postoperative recovery and allowed surgeons to become more comfortable with the STP concept. Third, there have been few complications and adverse effects, which has provided increasing evidence that patients who have undergone elective uneventful craniotomies do not necessarily require an ICU level of care postoperatively.

For institutions interested in adopting an STP for a subset of their craniotomy patients, we found it helpful and thus recommend enlisting a multidisciplinary and multidepartmental team approach at the beginning of the process, recognizing that changes in institutional culture often proceed slowly and require the support of executive leadership. Moreover, developing a pathway requires focused investigations into practice and protocol as well as critical evaluation of the inclusion population and ongoing re-evaluation to monitor safety, effectiveness, and any adverse events that may occur.

\section{Study Limitations}

There are a few limitations of the STP model to consider. First, the PACU is associated with high costs, and additional PACU time could generate expenses that offset some of the financial benefit resulting from bypassing ICU admission. Further evaluation is required to determine how a prolonged PACU stay can influence total cost savings. However, at some institutions patients who are assigned an ICU level of care may end up remaining in the PACU overnight only because there are no ICU beds available, which is very costly. The ability to downgrade these patients to a TCU level of care may actually result in shorter PACU stays. Second, the STP model results in less monitoring by nurses, which could theoretically put patients at risk immediately after surgery. Although rare, complications following cranial neurosurgery, including postoperative hematoma or seizure, could be devastating if the diagnosis is delayed. ${ }^{9,12,16}$ However, our study showed that there are few complications following surgery in ap- propriately selected patients and that they did not require elevation of care to the ICU level. As alluded to previously, recently reported studies have shown that same-day discharges for tumor resections and surgical clipping of unruptured cerebral aneurysms can be performed with minimal adverse outcomes. ${ }^{17-19}$ Third, direct admission to the NTCU could utilize all of the NTCU beds and actually delay the transfer of patients out of the ICU, which would theoretically keep more patients from being downgraded from ICU status and negate the savings made by avoiding the ICU admission in this STP cohort. However, if there are more beds in the NTCU than the ICU, a bottleneck effect is less likely to occur. Finally, subtle differences in patient satisfaction and readmission rates may have failed to reach significance due to the power of this study, which was limited by the sample size of the patient and nursing staff survey, and future work will continue to monitor these metrics for our patient ICU and NTCU pathway cohorts.

\section{Conclusions}

We found that admitting selected, generally otherwise healthy, postoperative craniotomy patients directly from the PACU to the step-down unit, bypassing the ICU, is safe, and, as one might expect, can result in cost savings and does not adversely affect the patient or provider experience. The cohort consisted of select patients who underwent supratentorial brain tumor resection, MVD, or Chiari malformation decompression. LOS, direct cost, and ICU days were significantly shorter in our STP cohort. There were no substantial patient complications. The STP may be a viable model for other institutions to adopt, to lower costs and decrease patient LOS.

\section{Acknowledgments}

This study was supported by a Caring Wisely grant from UCSF Medical Center.

\section{References}

1. Halpern NA. Can the costs of critical care be controlled? Curr Opin Crit Care. 2009;15(6):591-596.

2. Norris C, Jacobs P, Rapoport J, Hamilton S. ICU and nonICU cost per day. Can J Anaesth. 1995;42(3):192-196.

3. Curtis JR, Engelberg RA, Bensink ME, Ramsey SD. Endof-life care in the intensive care unit: can we simultaneously increase quality and reduce costs? Am J Respir Crit Care Med. 2012;186(7):587-592.

4. Kelly DF. Neurosurgical postoperative care. Neurosurg Clin N Am. 1994;5(4):789-810.

5. Awad IA. Intensive care after elective craniotomy: "all politics is local". World Neurosurg. 2014;81(1):64-65.

6. Hecht N, Spies C, Vajkoczy P. Routine intensive care unitlevel care after elective craniotomy: time to rethink. World Neurosurg. 2014;81(1):66-68.

7. Hanak BW, Walcott BP, Nahed BV, et al. Postoperative intensive care unit requirements after elective craniotomy. World Neurosurg. 2014;81(1):165-172.

8. Osorio JA, Safaee MM, Viner J, et al. Cost-effectiveness development for the postoperative care of craniotomy patients: a safe transitions pathway in neurological surgery. Neurosurg Focus. 2018;44(5):E19.

9. Wong JM, Panchmatia JR, Ziewacz JE, et al. Patterns in 
neurosurgical adverse events: intracranial neoplasm surgery. Neurosurg Focus. 2012;33(5):E16.

10. Steinwald B, Dummit LA. Hospital case-mix change: sicker patients or DRG creep? Health Aff (Millwood). 1989;8(2):3547.

11. Jackson C, Westphal M, Quiñones-Hinojosa A. Complications of glioma surgery. Handb Clin Neurol. 2016;134:201218.

12. Florman JE, Cushing D, Keller LA, Rughani AI. A protocol for postoperative admission of elective craniotomy patients to a non-ICU or step-down setting. J Neurosurg. 2017;127(6):1392-1397.

13. Venkatraghavan L, Bharadwaj S, Au K, et al. Same-day discharge after craniotomy for supratentorial tumour surgery: a retrospective observational single-centre study. Can J Anaesth. 2016;63(11):1245-1257.

14. Sughrue ME, Bonney PA, Choi L, Teo C. Early discharge after surgery for intra-axial brain tumors. World Neurosurg. 2015;84(2):505-510.

15. Richardson AM, McCarthy DJ, Sandhu J, et al. Predictors of successful discharge of patients on postoperative day 1 after craniotomy for brain tumor. World Neurosurg. 2019;126:e869-e877.

16. Mirian C, Møller Pedersen M, Sabers A, Mathiesen T. Antiepileptic drugs as prophylaxis for de novo brain tumourrelated epilepsy after craniotomy: a systematic review and meta-analysis of harm and benefits. J Neurol Neurosurg Psychiatry. 2019;90(5):599-607.

17. Carrabba G, Venkatraghavan L, Bernstein M. Day surgery awake craniotomy for removing brain tumours: technical note describing a simple protocol. Minim Invasive Neurosurg. 2008;51(4):208-210.

18. Goettel N, Chui J, Venkatraghavan L, et al. Day surgery craniotomy for unruptured cerebral aneurysms: a single center experience. J Neurosurg Anesthesiol. 2014;26(1):60-64.

19. Grundy PL, Weidmann C, Bernstein M. Day-case neurosurgery for brain tumours: the early United Kingdom experience. Br J Neurosurg. 2008;22(3):360-367.

\section{Disclosures}

Dr. A. K. Chan reports receiving support of a non-study-related clinical or research effort from Orthofix Inc. Dr. McDermott reports being a consultant for Stryker.

\section{Author Contributions}

Conception and design: McDermott, Viner, Sankaran, Imershein, Meary-Miller, Theodosopoulos, Jacques, Aghi, Chang,

Hervey-Jumper, T Ward, Gibson, M Ward, Sanftner, Wong, Amara, Osorio, Venkatesh, Gonzales, Lau, Boscardin, Wang, Berry, McCullagh, Reid, Reels, Nedkov, Berger. Acquisition of data: McDermott, Young, AK Chan, Viner, Imershein, T Ward, Venkatesh, Gonzales, Boscardin, Wang, Berry. Analysis and interpretation of data: McDermott, Young, AK Chan, Viner, Imershein, Amara, Magill, Osorio, Wang. Drafting the article: Young, AK Chan, Viner, AY Chan, Imershein, Magill. Critically revising the article: McDermott, Young, AK Chan, Viner, Sankaran, AY Chan, Imershein, Aghi, Chang, Hervey-Jumper, T Ward, Amara, Magill, Gonzales, Lau, Boscardin, Berger. Reviewed submitted version of manuscript: McDermott, Young, AK Chan, Viner, Sankaran, AY Chan, Imershein, Theodosopoulos, Jacques, Aghi, Chang, Hervey-Jumper, T Ward, Gibson, M Ward, Sanftner, Wong, Amara, Magill, Osorio, Venkatesh, Gonzales, Lau, Boscardin, Wang, Berry, McCullagh, Reid, Reels, Nedkov, Berger. Approved the final version of the manuscript on behalf of all authors: McDermott. Statistical analysis: Young, AK Chan, Viner, Imershein. Administrative/ technical/material support: Imershein, Meary-Miller, Venkatesh, Gonzales, Boscardin, Wang, Berry. Study supervision:

McDermott, Young, Berger.

\section{Correspondence}

Michael W. McDermott: University of California, San Francisco, CA.mcdermottm@neurosurg.ucsf.edu. 